\title{
Effect of squint surgery on pupillary diameter
}

\author{
C B James, J S Elston
}

\begin{abstract}
Aim-To investigate the effect that squint surgery has on pupillary diameter.

Methods-The effect of squint surgery on pupil size was investigated in 19 patients. Results-A significant mydriasis in the operated eye was observed when compared with the unoperated eye. This was independent of the number or type of extraocular muscles operated upon.

Conclusion-It is hypothesised that this change in pupillary diameter results from the release of neurotransmitters from tissues damaged during surgery.

(Br f Ophthalmol 1995; 79: 991-992)
\end{abstract}

The diameter of the pupils results from the interplay of sympathetic and parasympathetic influences. Both pupils are usually equal in diameter although anisocoria may occur in normal individuals. An induced anisocoria is seen in acute elevation of intraocular pressure, ocular inflammation, trauma, interruption of the nerves containing pupillomotor fibres, and after pharmacological intervention. ${ }^{1}$ Exposure to bright light ${ }^{2}$ and extreme lateral gaze (Tournay's phenomenon) have also been reported as causing uniocular mydriasis ${ }^{34}$ although these causes remain controversial. Anisocoria may also occur during routine squint surgery. The explanation for this phenomenon is unclear. It is possible that it results from the release of transmitters from tissues during surgery; alternatively, it may result from ischaemia to the iris following detachment of the rectus muscles. It is also possible that traction results in a form of Tournay's phenomenon. The following study was conducted to further investigate the effect that squint surgery has on pupillary diameter.

Oxford Eye Hospital, The Radcliffe Infirmary, Walton Street, Oxford OX2 6HE

C B James

J S Elston

Correspondence to: Mr Bruce James, Stoke Mandeville Hospital, Mandeville Road, Aylesbury, Bucks HP21 8AL.

Accepted for publication 15 June 1995

\section{Method}

Nineteen patients (13 males, six females, mean age 24.5 (SD $5 \cdot 1$ ) years) about to undergo squint surgery were studied. Six had undergone previous surgery for squint in the operated eye. None had any other ocular disease, in particular no history of previous pupillary abnormalities. All surgery was performed under general anaesthesia. The technique of anaesthesia and the anaesthetic agents used was not, however, uniform as it was dictated by the needs of the individual patient. One patient had only an inhalational anaesthetic, the remainder had a combination of intravenous and inhalational anaesthetic.

The following protocol was used to study each patient. Both eyes were photographed simultaneously at the start of surgery, just before placement of the stay sutures. A photograph was again taken following detachment of the first muscle and a final photograph was taken at the completion of surgery to the first eye. The muscles operated upon are shown in Table 1. Horizontal pupil diameter was measured with a pair of callipers on an image of the photograph projected onto a screen. Two pictures for two subjects recording pupil size at the end of surgery were of insufficient quality to permit accurate measurement. The position of the callipers was randomly reset between each measurement to overcome the possibility of observer bias as the side of the operation was apparent from the photographs. All measurements were made by the same person. A ratio of pupillary size in the operated to the unoperated eye was calculated to negate any change in the size of both pupils caused, for example, by the anaesthetic. The results were analysed using paired $t$ tests; differences between type and number of muscles operated upon were examined with unpaired $t$ tests.

\section{Results}

There was no statistically significant difference in the diameter of the pupils at the start of surgery (ratio operated to unoperated $=1.01$ (SD 0.01), $n=19$ ). After detachment of the first muscle a significant mydriasis occurred, the ratio of operated to unoperated pupil diameter increased significantly when compared with the control value $(1 \cdot 1(0 \cdot 03), \mathrm{p}<0.0001)$. Similarly the ratio of operated to unoperated pupil diameter remained significantly increased (1.09 $(0.03), p=0.008)$ at the end of surgery to the first eye. There was no apparent relation between the number of muscles operated on and the degree of mydriasis (difference in preoperative and end of operation ratio $=0.12$ $(0.04)$ for one muscle, $0.06(0.04)$ for two muscles, $\mathrm{p}=\mathrm{ns})$. Furthermore, mydriasis was seen with both the oblique and rectus muscle surgery (difference in preoperative and end of operation ratio $=0.08(0.03)$ for operations involving a rectus muscle and $0.1(0.08)$ for operations involving only an oblique muscle, $\mathrm{p}=\mathrm{ns})$. The maximum increase in pupil size was $33 \%$ in a patient who had a single medial rectus recession. Two patients showed a decrease in the pupil size in the operated eye of

Table 1 Details of the operations performed on the patients undergoing squint surgery

\begin{tabular}{ll}
\hline Number of subjects & Muscles involved \\
\hline 6 & Medial and lateral recti \\
4 & Medial rectus \\
3 & Inferior oblique \\
1 & Inferior rectus \\
2 & Inferior oblique, lateral rectus \\
1 & Superior and lateral recti \\
1 & Superior oblique, medial rectus \\
1 & Superior oblique, superior rectus \\
\hline
\end{tabular}


$1 \%$ and $5 \%$. Both these patients had horizontal recession/resections.

\section{Discussion}

A number of explanations for this induced anisocoria seen during squint surgery may be given. The mydriasis observed in this study in the operated eye during squint surgery may be attributed to the release of chemical messengers (adrenaline for example) from the traumatised conjunctiva or muscles. Venemans $e t a l^{5}$ have reported that the type of anaesthetic given plays a part in determining whether or not a pupil dilates. Anaesthetic agents might be expected to modify the response of the pupil to any chemical messenger. We did, however, see the phenomenon in patients receiving intravenous anaesthetic agent, which was not the case in the study of Venemans et al. It is, of course, possible that these agents may reduce the mydriatic response making its detection harder. Venemans et al used a measuring staff to record pupillary diameter.

The mydriasis may also be ascribed to a relative ischaemia of the iris resulting from detachment of the muscle from the sclera leading to the disruption of part of the anterior ciliary circulation. The latter explanation is unlikely; firstly, pupillary dilatation was observed in patients undergoing oblique muscle surgery which should not compromise the anterior ciliary vessels; secondly, there was no increased mydriasis in patients undergoing detachment of two muscles which might be expected to increase the disruption of the anterior ciliary circulation.

The traction of the eye into extreme positions of gaze may result in a form of Tournay's phenomenon although some controversy surrounds this phenomenon and indeed whether it occurs at all. ${ }^{34}$ It has been ascribed to a mechanical stretching of the nerves in the orbit on lateral gaze which may also occur during squint surgery. ${ }^{4}$ Not all surgery involved placing the eye in lateral gaze, however. Furthermore, Loewenfeld et al feel that a more likely explanation for the phenomenon is that there is an input to the Edinger Westphal nucleus from the supranuclear connections passing to the nucleus of the medial rectus. ${ }^{3}$

The light shining onto the eye during the operation may cause a relative dilatation of the pupil in the operated eye. High intensity light has been reported as causing a paradoxical pupillary dilatation in unanaesthetised monkeys. ${ }^{2}$ There may also be a temperature difference between the two eyes. It is, however, unlikely that any difference in either light or temperature would be great enough to cause a significant difference in pupil size. Similarly it is unlikely that there is a significant rise in intraocular pressure during surgery (to above the systolic ophthalmic artery pressure), a further cause of uniocular mydriasis. ${ }^{6}$

\section{Conclusion}

This study has shown that mydriasis occurs in the operated eye during squint surgery. The degree of mydriasis appears independent of the number and type of muscle operated upon. It is considered most likely to result from the release of chemical messengers from damaged tissues rather than ischaemia caused by disruption of the anterior ciliary blood vessels.

1 Miller NR. Walsh and Hoyt's clinical neuro-ophthalmology. Vol 2. 4th ed. Baltimore: Williams and Wilkins, 1985: 428-37.

2 Clarke AM, Behrendt T. Solar retinitis and pupillary reaction. Am $\mathcal{F}$ Ophthalmol 1972; 73: 700-3.

3 Loewenfeld IE, Friedlaender P, Mckinnon PFM. Pupillary inequality associated with lateral gaze (Tounay's phenominequality associated with lateral gaze (Tounay

4 Sharpe JA, Glaser JS. Tournay's phenomenon. Am $\mathfrak{f}$ Ophthalmol 1974; 77: 250-5.

5 Venemans EF, De Keizer RJW, Swart Van Den Berg M, Baartse WJ. Reversible mydriasis during strabismu surgery in children. Doc Ophthalmol 1993; 83: 65-70.

6 Rutkowski PC, Thompson S. Mydriasis and increased intraocular pressure. 1. Pupillographic studies. Arch Ophthalmol 1972; 87: 21-4. 